\title{
Téoros
}

Revue de recherche en tourisme

\section{La capitale et sa fonction touristique}

\section{Jacques Demers}

Volume 12, numéro 1, mars 1993

Québec, capitale et région touristiques

URI : https://id.erudit.org/iderudit/1078194ar

DOI : https://doi.org/10.7202/1078194ar

Aller au sommaire du numéro

Éditeur(s)

Université du Québec à Montréal

ISSN

0712-8657 (imprimé)

1923-2705 (numérique)

Découvrir la revue

Citer cet article

Demers, J. (1993). La capitale et sa fonction touristique. Téoros, 12(1), 7-9.

https://doi.org/10.7202/1078194ar d'utilisation que vous pouvez consulter en ligne.

https://apropos.erudit.org/fr/usagers/politique-dutilisation/ 


\title{
La capitale et sa fonction touristique
}

\author{
Jacques Demers*
}

$\square$

La notion de capitale demeure souvent un concept abstrait mais en pratique cette notion définit d'une façon plus systématique un centre urbain homogène ayant des caractéristiques distinctives par rapport à toute autre forme d'agglomération. Ces caractéristiques sont dues, pour une large part, à la présence du siège du gouvernement. Lorsqu'en 1608 Champlain arriva à Québec, les qualités du site lui sont apparues exceptionnelles, tout au moins sur le plan militaire. Le fleuve est étroit, le promontoire escarpé et la vue en aval permettait d'observer de loin toute manoeuvre ennemie. Au fil des ans, ce lieu a toujours abrité les fonctions politiques tant et si bien qu'en 1867, avec l'Acte de l'Amérique du Nord britannique, la ville de Québec devient le siège officiel du gouvernement. Àcette époque, la ville et la banlieue comptaient moins de 60000 habitants.

Bien que depuis plusieurs années on se préoccupe de mettre en valeur la capitale du Québec, ou tout au moins que plusieurs études ont fait l' objet de diverses recommandations en ce sens, ce n'est que récemment que le gouvernement créa le Secrétariat à la Capitale. Son mandat est précisément de coordonner les activités gouvemementales de façon à valoriser la ville et sa région.

Parmi tous les secteurs qui sont susceptibles d'influencer le profil de la Capitale, le tourisme occupe une place prépondérante. $\mathrm{Ce}$ pendant, n'étant pas la seule variable, il importe de l' intégrer harmonieusement dans le processus de façon à ce que son impact soit maximal.

Au cours des paragraphes qui suivent, il sera question, en premier lieu, des principaux critères d'authentification d'une capitale et de la relation du secteur tourisme avec l'ensemble du secteur manufacturier et des services. Par la suite, on verra les caractéristiques distinctives del' industrie touristiqueàQuébec comme secteur moteur.

\section{Quelques caractéristiques}

En premier lieu, il convient de s'attarder sur la dimension que peut avoir une capitale. Le gabarit, dans ce cas, nous semble avoir une valeur à la fois absolue et relative. D'une part, iln'estpasstrictementobligatoire qu'elle soit la plus importante agglomération d'un État, mais il est indispensable tout au moins qu'elle ait une assise démographique permettant une certaine autosuffisance et une rentabilité des équipements complémentaires à caractère socio-culturel. A ce titre, l'agglomération doit être en mesure de supporter certains équipements comme des salles de spectacles, des muscés, un centre de congrès de dimension respectable, une infrastructure de transport adéquate, etc.

Il est certain que l'importance démographique d'une agglomération constitue sans équivoque le premier critère qui la distingue. En se référant à une hiérarchie sommaire, on peut mentionner le hameau qui regroupe quelques maisons en milieu rural, puis le village où s' organise une vie communautaire plus élaborée. Déjà, il est difficile de déterminer d'une manière absolue le nombre d'habitants qui permet de passer au stade de village puis ensuiteàcelui de ville. Bien qu'il existe une relation entre le degré de l'organisation communautaire et l'importance de la population, le passage d'une catégorie à l'autre dépend en outre des besoins accrus du milieu, de motifs d'ordre politique (pouvoir d'emprunts par exemple) ou stratégique. À titre d'illustration, on peutidentifierdes villes de moins de 4000 habitants et des villages de plus de 8000 . En poursuivant cette hiérarchie de la ville, on passe à la métropole puis à la mégalopole.

Ainsi, du hameau à la mégalopole, chaque terme identifie dans l'esprit de chacun une dimension plus ou moins objective d'une agglomération. Mais lorsqu'on parle de capitale, il devient plus difficile de cerner une dimension puisqu'on se réfere non seulement au nombre d'habitants mais davantage à une fonction dominante au même titre que certaines villes à caractère résidentiel, industriel ou universitaire. C"est ainsi qu' une métropole ou une mégalopole peut être à la fois 
capitale mais pas nécessairement l'inverse (subtilité lexicologique, lorsqu'il s'agit de fonction, on utilise souvent le terme cité: Québec cité parlementaire).

Au-delà de toute cette sémantique, on constate que la notion de capitale est davantage subjective et que la distinction se manifeste par sa fonction qui détermine son contenu. En ce sens, dès l'instant où l'État fixe son siège social dans une ville en particulier, il lui confere inévitablement un nouveau profil.

Petit à petit se précisent alors certaines variables dominantes dues notamment à la présence de l'Administration publique, principal moteur de l'ćconomie. Par exemple, lesactivitéstertiaires dominent davantage a Québec; incidemment elles occupent environ $83 \%$ de la population. Loin d'être négative, l'Administration gouvernementale assure des revenus très stablesetrelativement élevés. D'autres domaines se développent plus spontanément, notamment le tourisme, les industries culturelles, le loisir en général.

Par contre, le secteur manufacturier ne se développe pas au même rythme que dans une métropole. À Québec, l'ensemble de ce secteur s'est quand même bien diversifié et se répartit dans une quarantaine d'espaces industriels bien localisés. Ainsi, dans la région de Québec, on dénombre 102 industries par 100000 habitants; ce qui correspond a lamoyenne des capitales canadiennesalors que dans les autres villes la moyenne est de 162.

N'empêcheque les services sontd'importants créateurs d'emplois mais il s'agit en grande partie d'emplois subordonnés, c'est-à-dire découlant directement de l'activité manufacturière. Selon Delmas, $430 \%$ des emplois dans les services dépendent directement de cette activité, la réciproque n'est que de $6 \%$. En conséquence, wla santé de $l^{\prime}$ industrie manufacturiere a donc un impact considérable sur celle des servicess.

Est-ce à dire que l'activité économique dans une capitale où la proportion d'entreprises manufacturières est ordinairement moins importante se retrouve dans une position de faiblesse? Nous aurions tendance à répondre par l'affirmative d'autant plus que la conjoncture économique des années 1990 tend à maintenir une stabilite relative des revenus. On n'a qu'à songer aux exigences de plus en plus réservées de la part des syndicats tant et si bien que parfois l'employé accepte le gel ou même une diminution de son salaire afin de préserver son emploi. $\AA$ cela, on peut ajouter la stagnation ou presque des emplois dans le secteur public. Mais cependant, la situation de Québec-Ville pernet d'être optimiste; on y reviendra.

Des solutions existent cependant et conviennent ordinairement bien dans le contexte économique d'une capitale. Par exemple, la rechercheetledéveloppement technologique sont des secteurs à privilégier dans le même sens qu'il convient de favoriser les secteurs d'activités qui créent des emplois dans leur propre sphère sans être subordonnés au secteur manufacturier. II s'agit précisément des loisirs, de la restauration et de l'hôtellerie, bref du secteur tourisme.

Eneffet, l'activité touristique est une industrie en soi qui se situe au niveau des services sans aucune dépendance formelle avecl'entreprise manufacturière. Par exemple, un parc industriel qui supporte un certain nombre d'emplois contribue à activer le commerce de détail de la région mais a peu de conséquence sur l'activité touristique. Pour mieux illustrer nos propos, prenons l'exempled'une destination touristique autonome comme les Bermudes où plus de $65 \%$ du PIB dépend des touristes. Il n'y existe aucune industrie manufacturière et le revenu per capita, en 1986, était de 18000 \$, bien au-delà des États-Unis (14461 \$) et des États américains à forte connotation manufacturière comme l'Ohio (13933 \$), l'Indiana (13 136\$) ou la Caroline du Nord (12 438 \$). Cet exemple, et il en existe plusieurs autres, illustre sans équivoque l'autarcie que peut susciter le secteur du tourisme, au même titre qu'une industrie primaire (bois, mines) peut faire vivre toute une population locale.

Ceci étant dit, on peut affirmer que Québec, la Capitale, se positionne avantageusement: une agglomération métropolitaine de l'ordre de 600000 personnes, la présence du siège du gouvernement et un potentiel touristique de premierplan. Nousavons là les principaux ingrédients pour que la Capitale occupe la place qui lui revient. Toutefois, encore fautil être conscient que la conjoncture politique et économique n'est plus celle des années 1970 , bien au contraire.

En réalité, nous nous acheminons vers une situation inédite où les gouvernements n'ont d'autre choix que d'équilibrer leurs déficits tout en limitant les prélèvements auprès des contribuables sachant fort bien que la creation d'emplois s'érode sous l'effet de la technologie. Ilconvientde soutenir l'industrie manufacturière dont la productivité supporte davantage le pouvoir d'achat et de developper plus agressivement le secteur des services non subordonnés et créateurs d'emplois, notamment l'industrie touristique. Examinons ce dernier aspect dans le contexte de la Capitale et sa région.

\section{La fonction touristique de la Capitale}

Il serait presomptueux de penser que par le simple fait d' être capitale, une ville attire les touristes. En réalité, nombreux sont ceux qui visitent Québec sans même se préoccuperdu fait qu'elle soit la capitale de la province. Même qu'un grand nombre l'ignore totalementquand on songeque $20 \%$ des Québécois ne le savent pas eux-même.

Par contre, il n'en demeure pas moins que sonprofil touristiqueest quelque peu façonne par laprésence du gouvernement. D'ailleurs, cetteprésences'exprime de plusieurs façons, non seulement par les édifices gouvernementaux, toujours majestueux, mais aussi par son environnement, son profil culturel, son histoire.

Parmi les valeurs patrimoniales, on songe sans hésiter à l' arrondissement historique, un sitede 135 hectares, correspondantaux limites de la vieille ville sous le Régime français et reconnue comme ville du patrimoinemondial. En dehors des fortifications, de nombreux monuments et plusieurs sites font l'orgueil de la ville. Mentionnons entre autres le parc des Champs-de-batailles, le parc CartierBrébeuf, celui des Chutes Montmorency et à plusieurs autres situés à l' intérieur des limites de la Communauté urbaine (CUQ).

Il faut mentionner la richesse touristique du fleuve Saint-Laurent dont le potentiel est à peine exploité. Outre les naviresde croisière, il existe près d'une dizaine de petits bateliers offrant diverses excursions. Sans doute que cette forme d'activité aurait avantage à être mieux connue, facilitant d'autant son expansion.

Un avantage comparatif unique consiste en la proximité des activités de plein air. $\AA$ moins d"une demi-heure du centre-ville, on accede à cinq stations de ski incluant leMont Sainte-Anne et Stoneham et en moins d'une heure a Petite-Rivière-Saint-François. Peu de capitales canadiennes peuvent se prévaloir de cette situation. Dans la même veine, le parc de la Jacques-Cartier à $35 \mathrm{~km}$ de 
Québec offre un site d'une remarquable beauté où plusieurs activités bien encadrées sont disponibles.

À cause précisément de ces ressources, la ville se situe parmi lesmeilleures destinations de congrès au Canada. Récemment, le gouvernement du Québec annonçait la construction d'un nouveau centre pouvant accueillir plus de 3000 congressistes etsituéa proximité de la Colline parlementaire, à deux pas du Vieux-Québec.

Outreces richessesreliées au potentiel naturel et à la situation géographique de la Capitale, d'autres valeurs émergent notamment de son profil culturel. En tant que siège du gouvernement, on note la présence d'un plus grand nombre d'institutions culturelles de l'État et de sièges sociaux d'organismes culturels a rayonnement national et intemational. Mentionnons, dans le cas de Québec, les Archives nationales du Québec, le Centre de conservation du Québec, les grands musées connus: le Musée du Québec, celui du Séminaire, le Musée de la civilisation, la Commission des biens culturels, l'Association des villes du patrimoine mondial, etc. Normalement, une capitale abrite la Bibliothèque nationale; celle du Québec est situcé àMontréalpour des raisons historiques. Sans entrer dans les détails, les arts de la scène sont aussi très présents en plus de nombreuses autres activités à caractère culturel: bibliothèques, expositions, événements.

Bienentendu, ledéveloppement cultureln'est pas nécessairement plus accentué que dans les autres villes d'importance mais il n'en demeure pas moins que certains volets sont plus développés alors que d'autres sont propres à la présence des institutions de l'État.

Une caractéristique incontestable est son passé historique. Bien qu'il existait déjà une petite communauté amérindienne sur le promontoire du Cap Diamant, bourgade qui s'appelait Stadaconé, comme on l'a tous appris, la naissance de l'Amérique française date officiellement de 1608 avec la fondation de Québec parChamplain. Si les circonstances ont voulu que Québec soit à l'origine de notre histoire et que la ville soit aussi la capitale, cela découle davantage de la convergence d'un ensemble de caractéristiques qui en ont fait un lieu privilégié pour l'administration gouvernementale. Par conséquent, aux caractéristiques plus conventionnelles s'ajoute la valeur historique et
Québec peut donc porter fièrement son titre de Vieille Capitale.

Au terme de cette brève réflexion, l'authenticité d'une capitale n'est pas virtuellement différente d'une autre ville, si ce n'est qu'elle est le siège du gouvernement. En apparence, rien ne la distingue à l'exception d'un ou quelques édifices parlementaires ordinairement importants et empreints de solennité. Cependant, dans les faits et intrinsèquement, elle differe, comme nous l'avons vu, sur plusieurs plans notamment le tourisme.

Néanmoins, à Québec, il ne faut pas sousentendre que l'industrie touristique de la région ait atteint son rythme de croisière. En réalité, il convient de poursuivre l'action engagée et, encore mieux, l'accélérer. D'ailleurs, il faut s'attendre à une progression beaucoup plus lente de l'emploi dans la fonction publique; ne serait-il pas sage alors d'accentuer le développement des secteurs manufacturieret touristique? Encoremieux, compte tenu du potentiel régional et de la qualité des services, le développement touristique y trouve un terrain propice à une expansion plus rapide par rapport aux autres secteurs. Aussi bien alors lui accorder la priorité.

Cependant, cela exige une complicitế entre les partenaires, notamment l'Association touristique régionale, le Conseil régional de développement, l'Office du tourisme et des congrès de laCUQ et le gouvernement. Àcet égard, on peut affirmer que là n'est pas la difficulté mais lorsqu'on songe que la ville de Québec n'est surtout pas une capitale régionale mais bien celle de toute la province, le consensus semble plus difficile. En effet, dans la mesure où chaque Québécois, qu'il soitdeHull, deMontréal, de Chicoutimi ou d'ailleurs, est véritablement conscient que la ville deQuébec est la Capitale del'État dans son ensemble, chacun doit au moins afficherun minimum de fierté, reconnaître la valeur de ce symbole et être favorable à son éclosion.

S'ilsembleévident queles touristesne visitent pas une ville parce qu'elle est une capitale mais parce que sous l'influence du contexte administratif gouvernemental, elle differe par son contenu, en contrepartie, ce contenu doit être à l'image de tout le Québec et non a celle d'une seule région. IIn'endemeure pas moins que Québec constitue une capitale exceptionnelle. Sans reprendre ses caracté- ristiques, on peut affirmer que son cachet, sa topographie, son environnement, son architecture, etc., seraient différents sans la présence du siège de l'Étatet sans doute elle ne serait pas une des plus belles villes au monde. 